\title{
FULL REGULARITY OF THE FREE BOUNDARY IN A BERNOULLI-TYPE PROBLEM IN TWO DIMENSIONS
}

\author{
Donatella Danielli and Arshak Petrosyan
}

\begin{abstract}
In this note we prove that in dimension $n=2$ there are no singular points on the free boundary $\partial\{u>0\} \cap \Omega$ in the Bernoulli-type problem governed by the $p$-Laplace operator$$
J_{p}(u)=\int_{\Omega}\left(|\nabla u|^{p}+\lambda_{p}^{p} \chi_{\{u>0\}}\right) d x \rightarrow \min ,
$$

for $p$ in the range $2-\varepsilon_{0}<p<\infty$ for an absolute constant $\varepsilon_{0}>0$.
\end{abstract}

\section{Introduction}

Let $u \geq 0$ be a bounded absolute minimizer of the energy functional

$$
J_{p}(v)=J_{p}(v ; \Omega):=\int_{\Omega}\left(|\nabla v|^{p}+\lambda_{p}^{p} \chi_{\{v>0\}}\right),
$$

where $\Omega \subset \mathbb{R}^{n}$ is a bounded open set, $1<p<\infty$, and $\lambda_{p}>0$ is a certain constant. This is understood in a sense that $u \in W^{1, p}(\Omega)$ and

$$
J_{p}(u) \leq J_{p}(v) \quad \text { whenever } u-v \in W_{0}^{1, p}(\Omega) .
$$

Then $u$ satisfies the following overdetermined Bernoulli-type problem

$$
\begin{aligned}
\Delta_{p} u:=\operatorname{div}\left(|\nabla u|^{p-2} \nabla u\right)=0 & \text { in }\{u>0\} \\
u=0, \quad|\nabla u|=c_{p} & \text { on } \partial\{u>0\} \cap \Omega
\end{aligned}
$$

in a certain generalized sense, where $c_{p}=\lambda_{p}(p-1)^{-1 / p}$. It is known that the free boundary

$$
\Gamma:=\partial\{u>0\} \cap \Omega
$$

is locally of finite Hausdorff $\mathcal{H}^{n-1}$ measure and the measure-theoretic reduced boundary $\Gamma_{\text {red }}:=\partial_{\text {red }}\{u>0\} \cap \Omega$ is a union of real-analytic hypersurfaces. Moreover, the conditions (1.2)-(1.3) are satisfied on $\Gamma_{\text {red }}$ in the classical sense. This result has been established by Alt and Caffarelli in their fundamental work [AC81] when $p=2$ and later generalized for all $1<p<\infty$ by the authors in [DP05].

It is also known that the set of singular points $\Gamma \backslash \Gamma_{\text {red }}$ is of $\mathcal{H}^{n-1}$ measure zero. On the other hand, it has been known since [AC81] (see also [Beu58]) that in dimension $n=2$ and when $p=2$ the free boundary is fully regular; i.e. it has no singular points.

Received by the editors August 23, 2005.

2000 Mathematics Subject Classification. Primary 35R35.

Key words and phrases. Full regularity, Free boundary problem, Bernoulli-type problem, $p$ Laplacian.

D. Danielli was supported in part by NSF grants DMS-0002801 and CAREER DMS-0239771.

A. Petrosyan was supported in part by NSF grant DMS-0401179. 
A natural question to ask, whether this result is true for all $1<p<\infty$. We give a partial answer to this question.

Main Theorem. Let $n=2$ and $u$ be an absolute minimizer of (1.1). Then the free boundary $\partial\{u>0\} \cap \Omega$ is real analytic if $2-\varepsilon_{0}<p<\infty$, where $\varepsilon_{0}>0$ is an absolute constant.

The method that we use goes back to Alt and Caffarelli [AC81]. Their approach is based on the following observation: if $u$ is an absolute minimizer of $J_{p}$ on every bounded domain in $\mathbb{R}^{n}$ and such that $|\nabla u|=c_{p}$ in $\{u>0\}$, then necessarily $u$ is a so-called halfspace solution; i.e. $u(x)=c_{p}\left(\left(x-x_{0}\right) \cdot e\right)_{+}$for some unit vector $e$ (see Lemma 4.2). One basically needs to show that any absolute minimizer is sufficiently close to a halfspace solution near any free boundary point $x_{0}$. The closeness is measured in terms of the functional

$$
\left.\frac{1}{r^{2}} \int_{B_{r}\left(x_{0}\right) \cap\{u>0\}}\left|c_{p}^{p}-\right| \nabla u\right|^{p} \mid d x
$$

Once we have that $u$ is sufficiently close to a halfspace solution as $r \rightarrow 0$, we apply the "flatness implies regularity" theorem to show that the free boundary is regular near $x_{0}$.

This method works very well for $p \geq 2$, but shows its limitations when $1<p<2$. Nevertheless, using a careful limiting procedure as $p \nearrow 2$ (see Lemmas 5.1 and 5.3) and a uniform-in- $p$ version of "flatness implies regularity" theorem (see Theorem 3.3) we were able to extend the result to the range $2-\varepsilon_{0}<p<2$ for some small $\varepsilon_{0}>0$.

When $p=2$, an alternative approach, based on a monotonicity formula, has been proposed by Weiss [Wei99], which allowed to reduce the question to the study of the existence of singular absolutely minimizing cones (i.e. homogeneous of degree one absolute minimizers). Taking this approach, Caffarelli, Jerison and Kenig [CJK04] established that there are no such cones when $n=3$, thus implying that the free boundary is fully regular in three dimensions. In contrast, De Silva and Jerison [DSJ05] showed the existence of a singular cone when $n=7$. These results draw a natural parallel with the theory of of minimal surfaces.

Unfortunately, no analogue of Weiss's monotonicity formula is known (at least to the authors at the time of writing) when $p \neq 2$. Having such a formula would trivialize the problem in dimension $n=2$, as it is elementary to show that there are no singular absolutely minimizing cones in the plane.

\section{Lipschitz continuity and nondegeneracy}

In the next two sections we recall some known properties of minimizers of the functional $J_{p}$ taken from [DP05]. In fact, we state the results in a slightly more general form, namely with constants depending uniformly on

$$
p \in I_{\mu}:=[1+\mu, 1+1 / \mu] \subset \subset(1, \infty), \quad \mu \in(0,1] .
$$

Since the regularity of the free boundary is a local property, we can restrict ourselves to the absolute minimizers of $J_{p}$ in balls centered at free boundary points. Also, in what follows we will normalize $\lambda_{p}=(p-1)^{1 / p}$ in (1.1) so that $c_{p}=1$ in (1.3).

Definition 2.1. Given $1<p<\infty$, and a ball $B_{\rho}\left(x_{0}\right)$, we say that $u \in S^{p}\left(B_{\rho}\left(x_{0}\right)\right)$ if 
(i) $u \geq 0$ in $B_{\rho}\left(x_{0}\right), \quad u \in W^{1, p}\left(B_{\rho}\left(x_{0}\right)\right) \cap L^{\infty}\left(B_{\rho}\left(x_{0}\right)\right)$;

(ii) $u$ is an absolute minimizer of the functional $J_{p}$ in (1.1) in $\Omega=B_{\rho}\left(x_{0}\right)$.

(iii) $x_{0} \in \partial\{u>0\}$.

Note that classes $S^{p}\left(B_{\rho}\left(x_{0}\right)\right)$ enjoy the following scaling and translating property:

$$
u \in S^{p}\left(B_{\rho}\left(x_{0}\right)\right) \Rightarrow u_{\lambda, x_{0}} \in S^{p}\left(B_{\rho / \lambda}\right),
$$

for any $\lambda>0$, where

$$
u_{\lambda, x_{0}}(x):=\frac{u\left(x_{0}+\lambda x\right)}{\lambda}, \quad x \in B_{\rho / \lambda} .
$$

Theorem 2.2 (Uniform Lipschitz continuity). Let $u \in S^{p}\left(B_{1}\right)$ with $p \in I_{\mu}$. Then there exists a constant $C=C(\mu, n)$ such that

$$
\|\nabla u\|_{L^{\infty}\left(B_{1 / 2}\right)} \leq C .
$$

Theorem 2.3 (Nondegeneracy). Let $u \in S^{p}\left(B_{1}\right)$ with $p \in I_{\mu}$. Then there exists $c=c(\mu, n)>0$ such that for $\gamma:=1+1 / \mu$

$$
\left(f_{B_{r}} u^{\gamma}\right)^{1 / \gamma} \geq c r, \quad 0<r \leq 1 / 2 .
$$

Theorem 2.4 (Density property). Let $u \in S^{p}\left(B_{1}\right)$ with $p \in I_{\mu}$. Then there exists a constant $c=c(\mu, n)>$ such that

$$
c \leq \frac{\left|\{u=0\} \cap B_{r}\right|}{\left|B_{r}\right|} \leq 1-c .
$$

These three theorems correspond to Theorem 3.3, Lemma 4.2, and Theorem 4.4 in [DP05], respectively. The only difference is that we now allow $p$ to change in $I_{\mu} \subset \subset(1, \infty)$. Therefore we will simply indicate the changes that are necessary to make in the corresponding proofs in [DP05]. Generally, the parts of the proof that are based on energy methods will go through with only slight cosmetic changes. However, the arguments that are based on the compactness methods will need special attention.

We start with uniform-in- $p$ versions of some known results for $p$-harmonic functions.

Lemma 2.5. Let $u$ be a bounded solution of $\Delta_{p} u=0$ in the unit ball $B_{1}$ in $\mathbb{R}^{n}$ with $p \in I_{\mu}$. Then there exists $\alpha=\alpha(\mu, n)$ such that

$$
\|u\|_{C^{1, \alpha}\left(B_{1 / 2}\right)} \leq C\left(\|u\|_{L^{\infty}\left(B_{1}\right)}, \mu, n\right) .
$$

Proof. This estimate is well known for fixed $1<p<\infty$. In particular, we refer to the papers of Evans [Eva82] (for $p \geq 2$ ) and Lewis [Lew83] (for $1<p \leq 2$ ), where one can easily trace that the constants depend uniformly on $p$ away from 1 and $\infty$.

Remark 2.6. In the special case $n=2$ that we consider in this paper, a direct proof can be given as follows. Without loss of generality we can assume that $u \in C_{\text {loc }}^{1, \beta}\left(B_{1}\right)$ with $\beta=\beta(p, n)$. For any unit vector $e$, the directional derivative $v:=u_{e}$ satisfies a uniformly elliptic equation in divergence form

$$
\sum_{i, j=1}^{n}\left(a_{i j}(x) v_{x_{i}}\right)_{x_{j}}=0, \quad a_{i j}(x):=\delta_{i j}+(p-2) \frac{u_{x_{i}} u_{x_{j}}}{|\nabla u|^{2}}
$$


in the region $\{|\nabla u|>0\}$, where $u$ is actually $C^{\infty}$. The ellipticity of $a_{i j}$ depends only on $\mu$ for $p \in I_{\mu}$; more specifically,

$$
\mu|\xi|^{2} \leq a_{i j}(x) \xi_{i} \xi_{j} \leq \mu^{-1}|\xi|^{2} .
$$

Using now the fact (which is known only for $n=2$ ) that the singular set $\{|\nabla u|=0\}$ is discrete [Man88] together with the local boundedness of $|\nabla u|$ we can remove the singularities of $v=u_{e}$, thus obtaining that (2.1) is satisfied in the entire ball $B_{1}$. Then, by the classical theorem of De Giorgi,

$$
\|v\|_{C^{\alpha}\left(B_{1 / 2}\right)} \leq C(\mu)\|v\|_{L^{2}\left(B_{3 / 4}\right)}
$$

and combining with Moser's, Hölder's, and Caccioppoli's inequalities

$$
\begin{aligned}
\|v\|_{L^{2}\left(B_{3 / 4}\right)} & \leq C_{1}(\mu)\|v\|_{L^{1+\mu}\left(B_{7 / 8}\right)} \\
& \leq C_{2}(\mu)\|v\|_{L^{p}\left(B_{7 / 8}\right)} \leq C_{3}(\mu)\|u\|_{L^{p}\left(B_{1}\right)} .
\end{aligned}
$$

This completes the proof of the lemma.

We also point out that in the case $n=2$ Manfredi [Man88] showed that one can actually take $\alpha=\mu$. Interestingly, $p$-harmonic functions in the plane enjoy much higher regularity when $p \rightarrow 1$, as shown by Iwaniec and Manfredi [IM89], however it is impossible to trace the constants in terms of $\|u\|_{L^{\infty}\left(B_{1}\right)}$.

Lemma 2.7 (Harnack inequality). Let $u \geq 0$ satisfy $\Delta_{p} u=0$ in $B_{1}$ with $p \in I_{\mu}$. Then there exists $C=C(\mu, n)$ such that

$$
\sup _{B_{1 / 2}} u \leq C \inf _{B_{1 / 2}} u .
$$

Proof. We refer to Theorem 1.1 in Trudinger [Tru67].

Lemma 2.8 (Weak Harnack inequality). Let $u \geq 0$ satisfy $\Delta_{p} u \geq 0$ in $B_{1}$ with $p \in I_{\mu}$. Then for $\gamma:=1+1 / \mu$ there exists $C=C(\mu, n)$ such that

$$
\sup _{B_{1 / 2}} u \leq C\left(\int_{B_{1 / 2}} u^{\gamma} d x\right)^{1 / \gamma} .
$$

Proof. We refer to Theorem 1.3 in Trudinger [Tru67].

The next lemmas are the essential steps in the proof of Theorems 2.2-2.3.

Lemma 2.9. Let $u \in S^{p}\left(B_{1}\right)$ with $p \in I_{\mu}$. Then there exists $\alpha=\alpha(\mu, n)$ such that

$$
\|u\|_{C^{\alpha}\left(B_{7 / 8}\right)} \leq C\left(\|u\|_{L^{\infty}\left(B_{1}\right)}, \mu, n\right)
$$

Proof. This lemma corresponds to Lemma 3.1 in [DP05] and requires no changes in the proof.

Lemma 2.10. Let $u \in S^{p}\left(B_{1}\right)$ with $p \in I_{\mu}$. Then

$$
\|u\|_{L^{\infty}\left(B_{1 / 4}\right)} \leq C(\mu, n) .
$$


Proof. We refer to the proof of Lemma 3.2 in [DP05]. Since the proof is by compactness, it requires some small changes that we outline below.

Arguing by contradiction, we assume that we there exists a sequence $u \in S_{p_{k}}\left(B_{1}\right)$, $k=1,2, \ldots$, for some $p_{k} \in I_{\mu}$ such that

$$
\max _{\bar{B}_{1 / 4}} u_{k}(x)>k .
$$

The main difference with Lemma 3.2 in [DP05] is that $p_{k}$ is now allowed to vary. Following the proof in [DP05] we construct the functions $w_{k}$ and $v_{k}$ exactly in the same way. Observe that using the uniform-in- $p$ version of the Harnack inequality (Lemma 2.7 above) the property (3.7) in [DP05] will be satisfied with a constant $c$ independent of $p_{k}$. Next, the equation (3.9) in [DP05], which now takes the form

$$
\int_{B_{3 / 4}}\left|\nabla\left(w_{k}-v_{k}\right)\right|^{p_{k}} d x \rightarrow 0
$$

easily implies by the Hölder inequality that

$$
\int_{B_{3 / 4}}\left|\nabla\left(w_{k}-v_{k}\right)\right|^{1+\mu} d x \rightarrow 0 .
$$

Assuming now that $p_{k} \rightarrow p_{0} \in I_{\mu}$, we can extract a subsequence such that $v_{k} \rightarrow v_{0}$ in $C^{1, \alpha}$-norm in $B_{5 / 8}$ (by Lemma 2.5 above). We thus have $\Delta_{p_{0}} v_{0}=0$ in $B_{5 / 8}$ in the distributional sense, since

$$
0=\int_{B_{5 / 8}}\left|\nabla v_{k}\right|^{p_{k}-2} \nabla v_{k} \cdot \nabla \eta d x \rightarrow \int_{B_{5 / 8}}\left|\nabla v_{0}\right|^{p_{0}-2} \nabla v_{k} \cdot \nabla \eta d x, \quad \eta \in C_{0}^{\infty}\left(B_{5 / 8}\right) .
$$

On the other hand, $w_{k}$ can be assumed to converge in $C^{\alpha}$ norm to some function $w_{0}$ (by Lemma 2.9 above). It follows from $(2.2)$ that $\nabla\left(w_{0}-v_{0}\right)=0$ in the distributional sense, thus implying $w_{0}=v_{0}+$ const in $B_{5 / 8}$. In particular we obtain that $w_{0}$ is $p_{0}$-harmonic. On the other hand $w_{0} \geq 0, w_{0}(0)=0$, and $\sup _{B_{1 / 2}} w_{0} \geq c>0$, which follows from the respective properties of the functions $w_{k}$. This, however, contradicts the strong minimum principle for $p_{0}$-harmonic functions.

The proof of the lemma is complete.

Proof of Theorems 2.2-2.4. The proofs of Theorems 2.2-2.3 follow from Lemmas 2.52.10 exactly in the same manner as the proofs of Theorem 3.3 and Lemma 4.2 in [DP05] do from their respective analogues. The proof of Theorem 2.4 follows the lines of the proof of Theorem 4.4 in [DP05], which uses a compactness arguments. Nevertheless it can be generalized to the case of variable $p$ precisely in the same way as in the proof of Lemma 2.10 .

\section{Flatness implies regularity}

In this section we recall the results from [DP05] concerning the regularity of the free boundary. As in the previous section we state the result in uniform-in- $p$ form and indicate how to obtain their proofs from the corresponding results in [DP05]. 
Definition 3.1. Let $0 \leq \sigma_{+}, \sigma_{-} \leq 1$ and $\tau>0$. We say that $u$ is of the flatness class $F^{p}\left(\sigma_{+}, \sigma_{-} ; \tau\right)$ in the ball $B_{\rho}$ if $u \in S^{p}\left(B_{\rho}\right)$ and

$$
\begin{array}{ll}
u(x)=0 & \text { for } x_{n} \geq \sigma_{+} \rho, \\
u(x) \geq-\left(x_{n}+\sigma_{-} \rho\right) & \text { for } x_{n} \leq-\sigma_{-} \rho, \\
|\nabla u| \leq 1+\tau & \text { in } B_{\rho}
\end{array}
$$

More generally, changing the direction $e_{n}$ by $\nu$ and the origin by $x_{0}$ in the definition above, we obtain definition of the flatness class $F^{p}\left(\sigma_{+}, \sigma_{-} ; \tau\right)$ in $B_{\rho}\left(x_{0}\right)$ in direction $\nu$.

Theorem 3.2 (Gradient Hölder estimate). Let $u \in S^{p}\left(B_{1}\right)$ with $p \in I_{\mu}$. Then there exist $C=C(\mu, n)>0$ and $\alpha(\mu, n)>0$ such that

$$
\sup _{B_{r}}|\nabla u| \leq 1+C r^{\alpha}, \quad 0<r<1 / 4 .
$$

Proof. This is the counterpart of Theorem 7.1 in [DP05]. The proof is based on the uniform gradient estimate (Lemma 2.2) and the fact that the $p$-Laplace equation $\Delta_{p} u=0$ can be written in a nondivergence form

$$
\sum_{i, j=1}^{n} a_{i j}(x) u_{x_{i} x_{j}}=0, \quad a_{i j}(x):=\delta_{i j}+(p-2) \frac{u_{x_{i}} u_{x_{j}}}{|\nabla u|^{2}},
$$

in $\{|\nabla u|>0\}$ where the ellipticity of the matrix $a_{i j}$ is uniform for $p \in I_{\mu}$ :

$$
\mu|\xi|^{2} \leq \sum_{i, j=1}^{n} a_{i j}(x) \xi_{i} \xi_{j} \leq(1 / \mu)|\xi|^{2} .
$$

Theorem 3.3 (Flatness implies regularity). Let $u \in S^{p}\left(B_{1}\right)$ with $p \in I_{\mu}$. Then there exist positive constants $\alpha, \beta, \sigma_{0}, \tau_{0}$ depending only on $n$ and $\mu$ such that

$$
\begin{aligned}
& \text { if } u \text { is of class } F^{p}(\sigma, 1 ; \infty) \text { in } B_{\rho}, \text { with } \sigma \leq \sigma_{0}, \rho \leq \tau_{0} \sigma^{2 / \beta} \text {, } \\
& \text { then } \partial\{u>0\} \cap B_{\rho / 4} \text { is a } C^{1, \alpha} \text { surface. }
\end{aligned}
$$

Proof. This is the analogue of Theorem 9.1 in [DP05] and Theorem 8.1 in [AC81]. The proof is obtained by iteration from Lemma 3.4 below, which corresponds to Lemma 7.10 in [AC81].

Lemma 3.4. Let $u \in S^{p}\left(B_{1}\right)$ with $p \in I_{\mu}$ and $\theta>0$. Then there exist constants $\sigma_{\theta}=\sigma(\theta, \mu, n)>0, c_{\theta}=c(\theta, \mu, n)>0$ and $C=C(n, \mu)>0$ such that if

$$
u \text { is of class } F^{p}(\sigma, 1 ; \tau) \text { in } B_{\rho} \text { in direction } \nu \text {, }
$$

with $\sigma \leq \sigma_{\theta}$ and $\tau \leq c_{\theta} \sigma^{2}$, then

$$
u \text { is of class } F^{p}\left(\theta \sigma, \theta \sigma ; \theta^{2} \tau\right) \text { in } B_{\bar{\rho}} \text { in direction } \bar{\nu}
$$

for some $\bar{\rho}, \bar{\nu}$ with $c_{\theta} \rho \leq \bar{\rho} \leq \rho / 4$ and $|\nu-\bar{\nu}| \leq C \sigma$. 
Proof. The proof is obtained by using the properties of the so-called nonhomogeneous blowup, see Section 8 in [DP05] (see also Section 7 in [AC81]). By using the uniformin- $p$ versions of the properties of absolute minimizers from our Section 2 as well as Lemmas 3.5-3.6 below, we realize that constants in the results of Section 8 in [DP05] depend uniformly on $p \in I_{\mu}$. We explicitly observe that even though it is a compactness argument, the proof doesn't change even if allow $p$ to vary.

Lemma 3.5. Let $u \in S^{p}\left(B_{1}\right)$ with $p \in I_{\mu}$. Then there exist $\sigma_{0}>0$ and $C_{0}>0$ depending only on $\mu$ and $n$ such that

$u$ is of class $F^{p}(\sigma, 1 ; \sigma)$ in $B_{1} \Longrightarrow u$ is of class $F^{p}\left(2 \sigma, C_{0} \sigma ; \sigma\right)$ in $B_{1 / 2}$

for $0<\sigma<\sigma_{0}$.

Lemma 3.6. Let $u \in S^{p}\left(B_{1}\right)$ with $p \in I_{\mu}$, and $\delta>0$. Then there exist $\sigma_{\delta}>0$ and $C_{\delta}>0$ depending only on $\delta, \mu$ and $n$ such that

$u$ is of class $F^{p}(\sigma, 1 ; \sigma)$ in $B_{1} \Longrightarrow|\nabla u| \geq 1-\delta$ in $B_{1 / 2} \cap\left\{x_{n} \leq-C_{\delta} \sigma\right\}$

for $0<\sigma<\sigma_{\delta}$.

Proof of Lemmas 3.5-3.6. See Theorems 6.3-6.4 in [DP05]. The proofs of these theorems in [DP05] are in some sense simultaneous and are obtained by bootstrapping from their weaker versions (see Lemmas 6.5-6.6 in [DP05]). Again, if we use the results stated in our Section 2, we will obtain the proof of Lemmas 3.5-3.6.

\section{Blowups and halfspace solutions}

The proof of Main Theorem that we will give in the next section will require the properties of so-called blowups with variable $p$. Namely, let $u_{k} \in S^{p_{k}}\left(B_{1}\right)$ with $p_{k} \in I_{\mu}, k=1,2, \ldots$, and consider the rescalings

$$
v_{k}(x)=u_{k, \lambda_{k}}(x):=\frac{u_{k}\left(\lambda_{k} x\right)}{\lambda_{k}}, \quad \text { for } x \in B_{1 / \lambda_{k}}
$$

for a certain sequence $\lambda_{k} \rightarrow 0$. Since $\nabla v_{k}(x)=\nabla u_{k}\left(\lambda_{k} x\right)$, by the uniform gradient bound in Theorem 2.2 we can assume that over a subsequence $v_{k}$ is converging in $C_{\text {loc }}^{\alpha}\left(\mathbb{R}^{n}\right)$ to a function $v_{0}$. We will call such $v_{0}$ a blowup.

Lemma 4.1. Let $v_{k} \in S^{p_{k}}\left(B_{R_{k}}\right)$ with $p_{k} \in I_{\mu}, p_{k} \rightarrow p_{0}$ and $R_{k} \rightarrow \infty$. Then there exist a subsequence (still denoted by $v_{k}$ ) and a function $v_{0}$, Lipschitz continuous in $\mathbb{R}^{n}$, such that

$$
\begin{array}{ll}
v_{k} \rightarrow v_{0} & \text { in } C_{\mathrm{loc}}^{\alpha}\left(\mathbb{R}^{n}\right) \\
\nabla v_{k} \rightarrow \nabla v_{0} & \text { a.e. in } \mathbb{R}^{n}
\end{array}
$$

for a certain Lipschitz continuous $v_{0}$ in $\mathbb{R}^{n}$. Then

$$
v_{0} \in S^{p_{0}}\left(B_{R}\right) \quad \text { for any } R>0 .
$$

Proof. By Theorem 2.2, after rescaling, we will have that $\left|\nabla v_{k}\right| \leq C=C(\mu, n)$ in $B_{R_{k} / 2}$. Thus, we can indeed extract a subsequence converging in $C_{\mathrm{loc}}^{\alpha}$ to a nonnegative Lipschitz continuous function $v_{0}$ in $\mathbb{R}^{n}$. Besides, we can assume that $\nabla v_{k} \rightarrow \nabla v_{0} *-$ weakly in $L_{\text {loc }}^{\infty}\left(\mathbb{R}^{n}\right)$.

Claim 1. Over a subsequence, $\nabla v_{k} \rightarrow \nabla u_{0}$ a.e. in $\mathbb{R}^{n}$. 
Indeed, let $x_{0} \in\left\{v_{0}>0\right\}$. Then there exist small $\gamma, \delta>0$ such that $v_{0} \geq \gamma$ in $B_{\delta}\left(x_{0}\right)$. Then, for sufficiently large $k, v_{k} \geq \gamma / 2$ in $B_{\delta}\left(x_{0}\right)$. In particular $v_{k}$ is $p_{k}$-harmonic in $B_{\delta}\left(x_{0}\right)$. Besides, $\left\{u_{k}\right\}$ is uniformly bounded in $B_{\delta}\left(x_{0}\right)$ and thus the uniform-in- $p$ interior $C^{1, \alpha}$ estimates (Lemma 2.5) yield that, over a subsequence, $\nabla v_{k} \rightarrow \nabla v_{0}$ uniformly in $B_{\delta / 2}\left(x_{0}\right)$. As a consequence, in order to prove the claim it remains to show that

$$
\nabla v_{k} \rightarrow \nabla v_{0} \quad \text { a.e. on }\left\{v_{0}=0\right\} \text {. }
$$

In the set $\left\{v_{0}=0\right\}$ a.a. points $x_{0}$ have density 1 . Denote the set of such points by $S$. We assert that if $x_{0} \in S$ then

$$
v_{0}\left(x_{0}+x\right)=o(|x|) .
$$

Assuming the contrary, let $r_{j} \rightarrow 0$ and $y_{j} \in B_{r_{j}}\left(x_{0}\right)$ be such that $v_{0}\left(y_{j}\right) \geq \gamma r_{j}$ for some $\gamma>0$. Then, by the Lipschitz continuity of $v_{0}$,

$$
v_{0}>\frac{\gamma}{2} r_{j} \quad \text { in } B_{c \gamma r_{j}}\left(y_{j}\right)
$$

for some $c>0$. This implies that $\left\{v_{0}>0\right\}$ has a positive density at $x_{0}$, contradicting $x_{0} \in S$.

From (4.1) we deduce that for any $\varepsilon>0$

$$
\frac{v_{k}}{r}<\varepsilon \quad \text { in } B_{r}\left(x_{0}\right) \text { for small } r \text {. }
$$

But then, by the uniform-in- $p$ nondegeneracy (Theorem 2.3), it follows that $v_{k}=0$ in $B_{r / 2}\left(x_{0}\right)$. Consequently $v_{0}=0$ in a neighborhood of $x_{0}$. This implies that $S$ is an open set. Furthermore, the above argument shows that $v_{k}=v_{0}=0$ on compact subsets of $S$ for sufficiently large $k$. This completes the proof of the claim.

Claim 2. $v_{0} \in S^{p_{0}}\left(B_{R}\right)$ in any $R>0$.

Let $\eta \in C_{0}^{\infty}\left(B_{R}\right)$ be such that $0 \leq \eta \leq 1$. Then for any $w_{0}$ with $w_{0}-v_{0} \in C_{0}^{\infty}\left(B_{R}\right)$ set

$$
w_{k}=w_{0}+(1-\eta)\left(v_{k}-v_{0}\right) .
$$

Therefore, $w_{k}=v_{k}$ on $\partial B_{R}$ and if $R_{k} \geq R$, we must have

$$
\int_{B_{R}}\left|\nabla v_{k}\right|^{p_{k}}+\lambda_{p_{k}}^{p_{k}} \chi_{\left\{v_{k}>0\right\}} \leq \int_{B_{R}}\left|\nabla w_{k}\right|^{p_{k}}+\lambda_{p_{k}}^{p_{k}} \chi_{\left\{w_{k}>0\right\}}
$$

Since $\left|\nabla v_{k}\right| \leq C$ and $\nabla v_{k} \rightarrow \nabla v_{0}$ a.e. by Claim 1 above,

$$
\int_{B_{R}}\left|\nabla v_{k}\right|^{p_{k}} \rightarrow \int_{B_{R}}\left|\nabla v_{0}\right|^{p_{0}}
$$

Similarly,

$$
\int_{B_{R}}\left|\nabla w_{k}\right|^{p_{k}} \rightarrow \int_{B_{R}}\left|\nabla w_{0}\right|^{p_{0}}
$$

Noting also that

we obtain from (4.2)

$$
\chi_{\left\{w_{k}>0\right\}} \leq \chi_{\left\{w_{0}>0\right\}}+\chi_{\{\eta<1\}},
$$

$$
\int_{B_{R}}\left|\nabla v_{0}\right|^{p_{0}}+\lambda_{p_{0}}^{p_{0}} \chi_{\left\{v_{0}>0\right\}} \leq \int_{B_{R}}\left|\nabla w_{0}\right|^{p_{0}}+\lambda_{p_{0}}^{p_{0}} \chi_{\left\{w_{0}>0\right\}}+\lambda_{p_{0}}^{p_{0}} \int_{B_{R}} \chi_{\{\eta<1\}} .
$$


Choosing a sequence of $\eta$ 's with $|\{\eta<1\}| \rightarrow 0$, we obtain that $v_{0}$ is an absolute minimizer of $J_{p_{0}}$ in $B_{R}$. Finally, to see that $0 \in \partial\left\{v_{0}>0\right\}$, observe that by the uniform-in- $p$ nondegeneracy (Theorem 2.3), after passing to the limit, we have

$$
\left(f_{B_{r}} v_{0}^{\gamma}\right)^{1 / \gamma}>c r, \quad r>0
$$

which implies that indeed $0 \in \partial\left\{v_{0}>0\right\}$.

Ultimately, we want to prove that all blowups $v_{0}$ are so-called halfspace solutions $v_{0}(x)=(x \cdot e)_{+}$for some unit vector $e$. We will use the following observation, which is the basis for Alt and Caffarelli's [AC81] approach.

Lemma 4.2 (Characterization of halfspace solutions). Let $v_{0}$ be as in Lemma 4.1 above; i.e. $v_{0} \in S^{p_{0}}\left(B_{R}\right)$ for any $R>0$. Suppose moreover

$$
\left|\nabla v_{0}\right|=1 \quad \text { a.e. in }\left\{v_{0}>0\right\} .
$$

Then there exists a unit vector e such that

$$
v_{0}(x)=(x \cdot e)_{+} \text {for any } x \in \mathbb{R}^{n} .
$$

Proof. Observe that $v_{0}$ is $p_{0}$-harmonic in $\left\{v_{0}>0\right\}$ so that $\left|\nabla v_{0}\right|=1$ actually at every point there. Let now $x_{0} \in\left\{v_{0}>0\right\}$. Then there exists a unit vector $e$ such that

$$
\partial_{e} v_{0}\left(x_{0}\right)=\left|\nabla v_{0}\left(x_{0}\right)\right|=1 \text {. }
$$

Consider now the partial derivative

$$
w(x):=\partial_{e} v_{0}(x)
$$

which satisfies a uniformly elliptic divergence-form equation

$$
\sum_{i, j=1^{n}}\left(a_{i j}(x) w_{x_{i}}\right)_{x_{j}}, \quad a_{i j}(x)=\delta_{i j}+\left(p_{0}-2\right) \frac{v_{0, x_{i}} v_{0, x_{j}}}{\left|\nabla v_{0}\right|^{2}}
$$

in $\left\{v_{0}>0\right\}$, since $\left|\nabla v_{0}\right|=1>0$ there. Now, by construction, we have $w\left(x_{0}\right)=1$. On the other hand

$$
w(x) \leq\left|\nabla v_{0}(x)\right|=1
$$

at every point in $\left\{v_{0}>0\right\}$. Thus, by the strict maximum principle applied to $w$, we obtain that $\partial_{e} v_{0}=1$ in the connected component of $\left\{v_{0}>0\right\}$. This also implies that $\partial_{\nu} v_{0}=0$ there for a direction $\nu$ orthogonal to $e$. Hence, $v_{0}(x)=((x \cdot e)-c)_{+}$in this connected component. Arguing in this way, we obtain that the only possibilities for $v_{0}$ are as follows (recall that $0 \in \partial\left\{v_{0}>0\right\}$ ):

1) $v_{0}(x)=(x \cdot e)_{+}$in $\mathbb{R}^{n}$, or

2) $v_{0}(x)=(x \cdot e)_{+}+(a-(x \cdot e))_{+}$in $\mathbb{R}^{n}$.

Let us show that the second case contradicts the fact that $v_{0} \in S^{p_{0}}(R)$ for large $R>0$. Indeed, we will easily have that

$$
\left|\left\{v_{0}=0\right\} \cap B_{R}\right| \leq C R^{n-1}
$$

while from the density property for the absolute minimizers (Theorem 2.4) we should have

$$
\left|\left\{v_{0}=0\right\} \cap B_{R}\right| \geq c R^{n}
$$

This is clearly a contradiction, which shows that the case 2$)$ is impossible. Hence $v_{0}(x)=(x \cdot e)_{+}$. The proof is complete. 
Using Lemmas 4.1-4.2 we can now state the conditions that guarantee that the blowup is a halfspace solution.

Lemma 4.3. Let $v_{k} \in S^{p_{k}}\left(R_{k}\right)$ with $p_{k} \in I_{\mu}, p_{k} \rightarrow p_{0}$, and $R_{k} \rightarrow \infty$. Suppose also that for a sequence $\varepsilon_{k} \rightarrow 0$ we have

(i) $\left|\nabla v_{k}\right| \leq 1+\varepsilon_{k}$ in $B_{R_{k}}$

(ii) for all $0<r<R_{k}$

$$
\frac{1}{r^{2}} \int_{B_{r} \cap\left\{v_{k}>0\right\}}\left(1-\left|\nabla v_{k}\right|^{p_{k}}\right) d x \leq \varepsilon_{k} .
$$

Then, there exists a unit vector e such that over a subsequence

$$
v_{k} \rightarrow(x \cdot e)_{+} \quad \text { in } C_{\mathrm{loc}}^{\alpha}\left(\mathbb{R}^{2}\right) .
$$

Proof. By Lemma 4.1, we can assume that $v_{k} \rightarrow v_{0}$ in $C_{\mathrm{loc}}^{\alpha}\left(\mathbb{R}^{2}\right)$, where $v_{0}$ is an absolute minimizer of $J_{2}$ in every ball $B_{R} \subset \mathbb{R}^{2}$. Moreover, we can assume that $\nabla v_{k} \rightarrow \nabla v_{0}$ a.e. in $\mathbb{R}^{2}$. In fact, we claim that there exists a unit vector $e$ such that

$$
v_{0}(x)=(x \cdot e)_{+} .
$$

First observe that $0 \in \partial\left\{v_{0}>0\right\}$, which follows from the uniform nondegeneracy of $v_{k}$ at $0 \in \partial\left\{v_{k}>0\right\}$, see Theorem 2.3. Next, because of a.e. convergence $\nabla v_{k} \rightarrow \nabla v_{0}$, conditions (i) and (ii) in the lemma will imply that

$$
\left|\nabla v_{0}\right|=1 \quad \text { a.e. in }\left\{v_{0}>0\right\} \text {. }
$$

Then by the characterization of halfspace solutions, see Lemma 4.2, $v_{0}(x)=(x \cdot e)_{+}$ in $\mathbb{R}^{2}$ for some unit vector $e$. The proof is complete.

\section{Full regularity of the free boundary}

In this section we prove Main Theorem.

The rough idea of the proof is as follows: we show that every $u \in S^{p}\left(B_{1}\right)$ is sufficiently close to a halfspace solution in a small neighborhood of the origin and therefore is flat. Then by Theorem 3.3 the free boundary is smooth near the origin.

To show the closeness to a halfspace solution, we use the characterization found in Lemmas 4.2-4.3. The analogue of the following core lemma in the case $p=2$ is due to Alt and Caffarelli [AC81].

Lemma 5.1. Let $n=2$ and $u \in S^{p}\left(B_{1}\right)$. Then

where

$$
\limsup _{r \searrow 0} \frac{1}{r^{2}} \int_{B_{r} \cap\{u>0\}}\left(1-|\nabla u|^{p}\right) d x \leq \gamma(p)
$$

$$
\begin{array}{ll}
\gamma(p)=0 & \text { for } p \geq 2 \\
\gamma(p) \rightarrow 0 & \text { as } p \nearrow 2 .
\end{array}
$$

Moreover, the inequality is uniform in the sense that for every $\varepsilon>0$ there exists $r(\varepsilon, p)>0$ such that

$$
\frac{1}{r^{2}} \int_{B_{r} \cap\{u>0\}}\left(1-|\nabla u|^{p}\right) d x \leq \gamma(p)+\varepsilon
$$

for any $0<r<r(\varepsilon, p), u \in S^{p}\left(B_{1}\right)$. 
Proof. Let $\zeta \in C_{0}^{\infty}\left(B_{1 / 2}\right), \zeta \geq 0$ and $\varepsilon>0$. Then

$$
u_{\varepsilon}:=\max (u-\varepsilon \zeta, 0)
$$

is an admissible function, and we have

$$
J_{p}(u) \leq J_{p}\left(u_{\varepsilon}\right)
$$

Thus,

$$
\begin{aligned}
\int_{\{0<u \leq \varepsilon \zeta\}} \lambda_{p}^{p} d x & \leq \int_{B_{1}}\left(\left|\nabla u_{\varepsilon}\right|^{p}-|\nabla u|^{p}\right) d x \\
& =-\int_{\{0<u \leq \varepsilon \zeta\}}|\nabla u|^{p} d x+\int_{\{u>\varepsilon \zeta\}}\left(\left|\nabla u_{\varepsilon}\right|^{p}-|\nabla u|^{p}\right) d x .
\end{aligned}
$$

To estimate the second integral, we use the inequality

$$
|\xi|^{p}-|\eta|^{p} \leq-p|\xi|^{p-2} \xi \cdot(\xi-\eta)
$$

for any $\xi, \eta \in \mathbb{R}^{n}$ and $p>1$, which follows from the convexity of the function $\xi \mapsto|\xi|^{p}$. Using also that $\Delta_{p} u=0$ in $\{u>0\}$ we will obtain

$$
\begin{aligned}
\int_{\{0<u \leq \varepsilon \zeta\}} \lambda_{p}^{p} d x \leq & -\int_{\{0<u \leq \varepsilon \zeta\}}|\nabla u|^{p} d x-p \int_{\{u>\varepsilon \zeta\}}\left|\nabla u_{\varepsilon}\right|^{p-2} \nabla u_{\varepsilon} \cdot \nabla(\varepsilon \zeta) d x \\
& +p \int_{\Omega}|\nabla u|^{p-2} \nabla u \cdot \nabla \min (\varepsilon \zeta, u) d x \\
= & (p-1) \int_{\{0<u \leq \varepsilon \zeta\}}|\nabla u|^{p} d x \\
& +p \int_{\{u>\varepsilon \zeta\}}\left(|\nabla u|^{p-2} \nabla u-\left|\nabla u_{\varepsilon}\right|^{p-2} \nabla u_{\varepsilon}\right) \cdot \nabla(\varepsilon \zeta) d x .
\end{aligned}
$$

Now, recall that we normalized $\lambda_{p}=(p-1)^{1 / p}$. Thus, we will have

$$
\begin{aligned}
\int_{\{0<u \leq \varepsilon \zeta\}} & \left(1-|\nabla u|^{p}\right) d x \leq \\
& \frac{p}{p-1} \int_{\{u>\varepsilon \zeta\}}\left(|\nabla u|^{p-2} \nabla u-\left|\nabla u_{\varepsilon}\right|^{p-2} \nabla u_{\varepsilon}\right) \cdot \nabla(\varepsilon \zeta) d x .
\end{aligned}
$$

To estimate the right-hand side in (5.3), we use the following inequality

$$
\left.|| \xi\right|^{p-2} \xi-|\eta|^{p-2} \eta \mid \leq\left\{\begin{array}{lr}
(p-1) \max \left(|\xi|^{p-2},|\eta|^{p-2}\right)|\xi-\eta| & \text { for } p \geq 2 \\
2^{2-p}|\xi-\eta|^{p-1} & \text { for } 1<p<2 .
\end{array}\right.
$$

for any $\xi, \eta \in \mathbb{R}^{n}$. The easy proof is left to the reader.

According to the cases in the inequality (5.4), we subdivide our proof into two parts.

Case 1: $p \geq 2$. Recall that by Theorem 2.2 we have $|\nabla u| \leq C(p)$ in $B_{1 / 2}$, which also implies that $u \leq C(p) r$ in $B_{r}$ for $r \leq 1 / 2$. Furthermore, arguing as in Theorem 6.6 
from [AC81], given small $0<r<R=1 / 2$, we choose $\varepsilon=C(p) r$ and

$$
\zeta(x)= \begin{cases}1 & \text { for } x \in B_{r}, \\ \frac{\log (R /|x|)}{\log (R / r)} & \text { for } x \in B_{R} \backslash B_{r}, \\ 0 & \text { elsewhere, }\end{cases}
$$

which is the 2-capacitary potential of the annular region $B_{R} \backslash \bar{B}_{r}$. Note that for small $r$ we will have $|\nabla(\varepsilon \zeta)| \leq C_{1}(p)$ in $B_{1}$ and consequently $\left|\nabla u_{\varepsilon}\right| \leq \max \left(C(p), C_{1}(p)\right)$. Then, from (5.3) and (5.4) it follows

$$
\begin{aligned}
\int_{\{0<u \leq \varepsilon \zeta\}}\left(1-|\nabla u|^{p}\right) d x & \leq p \int_{\{u>\varepsilon \zeta\}} \max \left(|\nabla u|^{p-2},\left|\nabla u_{\varepsilon}\right|^{p-2}\right)|\nabla(\varepsilon \zeta)|^{2} d x \\
& \leq C_{2}(p) \int_{\{u>\varepsilon \zeta\}}|\nabla(\varepsilon \zeta)|^{2} d x .
\end{aligned}
$$

Now observing that $B_{r} \subset\{u \leq \varepsilon \zeta\}$ and explicitly computing the 2-capacity of the ring $B_{R} \backslash B_{r}$, we will arrive at

$$
\int_{\{u>0\} \cap B_{r}}\left(1-|\nabla u|^{p}\right) d x \leq C_{3}(p) \frac{r^{2}}{\log (R / r)}
$$

and letting $r \rightarrow 0$, we conclude that

$$
\limsup _{r \rightarrow 0} \frac{1}{r^{2}} \int_{\{u>0\} \cap B_{r}}\left(1-|\nabla u|^{p}\right) d x \leq 0 .
$$

Case 2: $1<p<2$. The inequalities (5.3)-(5.4) imply in this case that

$$
\int_{\{0<u \leq \varepsilon \zeta\}}\left(1-|\nabla u|^{p}\right) d x \leq \frac{p 2^{2-p}}{p-1} \int_{\{u>\varepsilon \zeta\}}|\nabla(\varepsilon \zeta)|^{p} d x .
$$

Note that for the purpose of the lemma we may assume $p \in[3 / 2,2)$. Then by Theorem 2.2 there exists an absolute constant $C_{0}>0$ such that $|\nabla u| \leq C_{0}$ in $B_{1 / 2}$. Then for a given $0<r<R=1 / 2$, we choose $\varepsilon=C_{0} r$ and deduce

$$
\int_{\{0<u \leq \varepsilon \zeta\}}\left(1-|\nabla u|^{p}\right) d x \leq \frac{p 2^{2-p} C_{0}^{p}}{p-1} r^{p} \int_{\{u>\varepsilon \zeta\}}|\nabla \zeta|^{p} d x .
$$

Let now $\zeta(x)=\eta(x / r)$, where

$$
\eta(x)= \begin{cases}1 & \text { for } x \in B_{1}, \\ \frac{|x|^{-\alpha}-\rho^{-\alpha}}{1-\rho^{-\alpha}} & \text { for } x \in B_{\rho} \backslash B_{1}, \\ 0 & \text { elsewhere }\end{cases}
$$

for $\rho=R / r$ and

$$
\alpha=\alpha(p):=\frac{2-p}{p-1} .
$$


Notice that $\eta$ is the $p$-capacitary potential of the annular region $B_{\rho} \backslash \bar{B}_{1}$. Then,

$$
\begin{aligned}
r^{p} \int_{B_{R} \backslash B_{r}}|\nabla \zeta|^{p} d x & =r^{2} \int_{B_{\rho} \backslash B_{1}}|\nabla \eta|^{p} d x \\
& =2 \pi r^{2} \frac{\alpha^{p-1}}{\left(1-\rho^{-\alpha}\right)^{p-1}} \rightarrow 2 \pi r^{2} \alpha^{p-1} \quad \text { as } \rho \rightarrow \infty .
\end{aligned}
$$

Combining (5.7)-(5.8) and observing that $B_{r} \subset\{u \leq \varepsilon \zeta\}$, we obtain that

$$
\limsup _{r \rightarrow 0} \frac{1}{r^{2}} \int_{\{u>0\} \cap B_{r}}\left(1-|\nabla u|^{p}\right) d x \leq \frac{p 2^{2-p} C_{0}^{p}}{p-1}\left(\frac{2-p}{p-1}\right)^{p-1} .
$$

This completes the proof of the lemma.

We are now ready to prove the Main Theorem.

Proof of the Main Theorem. Without loss of generality we may assume that $u \in S^{p}\left(B_{1}\right)$ and we will need to show that $\partial\{u>0\} \cap B_{r}$ is a smooth surface for small $r>0$. The idea is to show that the statement in Lemma 5.1 implies that $u$ is of flatness class $F(\sigma, 1 ; \infty)$ in $B_{r}$ for sufficiently small $\sigma$, which by Theorem 3.3 will then imply the smoothness of the free boundary near the origin.

Case 1: $p \geq 2$. The proof of the Main Theorem in this case does not actually require the uniform-in- $p$ versions of the theorems that we stated earlier in the paper; the versions with fixed $p$ would be sufficient.

Lemma 5.2. Let $p \geq 2$. Then for any $\sigma>0$ there exists $\rho=\rho(\sigma, p)>0$ such that any $u \in S^{p}\left(B_{1}\right)$ is of flatness class $F(\sigma, 1 ; \infty)$ in $B_{\rho}$ in some direction $\nu$.

Proof. Assume the contrary. Then there exists $\sigma>0$ and sequences $\rho_{k} \rightarrow 0$ and $u_{k} \in S^{p}\left(B_{1}\right)$ such that $u_{k}$ is not of class $F(\sigma, 1 ; \infty)$ in any direction $\nu$ in $B_{\rho_{k}}$. Consider then

$$
v_{k}(x):=\frac{u_{k}\left(\rho_{k} x\right)}{\rho_{k}} \quad \text { in } B_{1 / \rho_{k}} .
$$

Then from Lemma 5.1 and Theorem 3.2, the sequence $v_{k}$ will satisfy the conditions of Lemma 4.3 with $p_{k}=p_{0}=p$. Hence, over a subsequence, $v_{k} \rightarrow(x \cdot e)_{+}$uniformly on every compact subset of $\mathbb{R}^{2}$. By the nondegeneracy (Theorem 2.3) we obtain that for sufficiently large $k, v_{k}$ must vanish on $B_{1} \cap\{x \cdot e \leq-\sigma\}$, implying that it is of the flatness class $F(\sigma, 1 ; \infty)$ in $B_{1}$. Rescaling back to $u_{k}$ we obtain that it is the flatness class $F(\sigma, 1 ; \infty)$ in $B_{\rho_{k}}$, contrary to our assumption. This completes the proof of the lemma.

When $p \geq 2$, the Main Theorem follows easily from Lemma 5.2 and Theorem 3.3. Indeed, let $p \geq 2$ be fixed and constants $\alpha, \beta, \sigma_{0}, \tau_{0}$ be as in Theorem 3.3. Then by Lemma 5.2 , there exists $\rho \leq \tau_{0} \sigma_{0}^{2 / \beta}$ such that $u$ is of flatness class $F\left(\sigma_{0}, 1 ; \infty\right)$ in $B_{\rho}$ in some direction $\nu$. Then, by Theorem 3.3, $\partial\{u>0\} \cap B_{\rho / 4}$ is a $C^{1, \alpha}$ surface and therefore smooth and this completes the proof of the Main Theorem when $p \geq 2$. 
Case 2: $p<2$. This case is trickier, and it is unknown to the authors if the statement of Lemma 5.2 is still valid for all $1<p<2$. However, if we have take $p$ sufficiently close to 2 we can guarantee the free boundary to be as flat as we wish.

Lemma 5.3. For every $\sigma \geq 0$ there exists an $\varepsilon_{0}=\varepsilon_{0}(\sigma)>0$ such that if $2-\varepsilon_{0}<$ $p<2$, then any absolute minimizer $u \in S^{p}\left(B_{1}\right)$ will be in the flatness class $F(\sigma, 1 ; \infty)$ in $B_{\rho(p)}$ in some direction $\nu$ and for some radius $\rho(p)>0$.

Proof. From Lemma 5.1, for every $1<p<2$, there exist $r(p)>0$ such that

$$
\frac{1}{r^{2}} \int\left(1-|\nabla u|^{p}\right) d x \leq 2 \gamma(p) \quad \text { for every } 0<r \leq r(p) \text { and } u \in S^{p}\left(B_{1}\right) .
$$

Besides, we can assume from Theorem 3.2 that

$$
|\nabla u| \leq 1+\varepsilon(p) \quad \text { in } B_{r(p)}
$$

for some $\varepsilon(p) \rightarrow 0$ as $p \nearrow 2$. We claim that one can take $\rho(p)=r(p)^{2}$ in the assertion of the lemma. Assuming the contrary, there exists a $\sigma>0$, a sequence $p_{k} \nearrow 2$ and minimizers of $u_{k} \in S^{p_{k}}\left(B_{1}\right)$ such that $u_{k}$ does not belong to the flatness class $F(\sigma, 1 ; \infty)$ in $B_{\rho(p)}$ in any direction $\nu$. Consider then the rescalings

$$
v_{k}(x)=\frac{u_{k}\left(\rho\left(p_{k}\right) x\right)}{\rho\left(p_{k}\right)} \quad \text { in } B_{1 / \sqrt{\rho\left(p_{k}\right)}}
$$

Assuming, without loss of generality, that $\rho(p) \rightarrow 0$ as $p \nearrow 2$, we notice that the sequence $v_{k}$ satisfies the conditions of Lemma 4.3 with $p_{0}=2$. As a consequence, over a subsequence, $v_{k} \rightarrow(x \cdot e)_{+}$uniformly on every compact subset of $\mathbb{R}^{2}$. By the uniform-in- $p$ nondegeneracy (Theorem 2.3) we obtain that for sufficiently large $k, v_{k}$ must vanish on $B_{1} \cap\{x \cdot e \leq-\sigma\}$, implying that it is of the flatness class $F(\sigma, 1 ; \infty)$ in $B_{1}$. Rescaling back to $u_{k}$ we obtain that it is the flatness class $F(\sigma, 1 ; \infty)$ in $B_{\rho\left(p_{k}\right)}$, contrary to our assumption. This completes the proof of the lemma.

When $p<2$, the Main Theorem follows easily from Lemma 5.3 and Theorem 3.3. Indeed, let the absolute constants $\alpha, \beta, \sigma_{0}, \tau_{0}$ be as in Theorem 3.3 with $\mu=1 / 2$. Then by Lemma 5.3 , there exists a small $\varepsilon_{0}>0$ such that every $u \in S^{p}\left(B_{1}\right)$ with $2-\varepsilon_{0}<p<2$ is of flatness class $F\left(\sigma_{0}, 1 ; \infty\right)$ in the ball $B_{\rho(p)}$ in some direction $\nu$. Without loss of generality we may assume that $\rho(p) \leq \tau_{0} \sigma_{0}^{2 / \beta}$. Then applying Theorem 3.3, we obtain that $\partial\{u>0\} \cap B_{\rho(p) / 4}$ is $C^{1, \alpha}$ and therefore smooth.

The proof of the Main Theorem is complete.

\section{References}

[AC81] H. W. Alt and L. A. Caffarelli, Existence and regularity for a minimum problem with free boundary, J. Reine Angew. Math. 325 (1981) 105-144.

[Beu58] A. Beurling, On free-boundary problems for the Laplace equations, Sem. Analytic Functions 1 (1958) 248-263.

[CJK04] L. A. Caffarelli, David Jerison, and Carlos E. Kenig, Global energy minimizers for free boundary problems and full regularity in three dimensions, Noncompact problems at the intersection of geometry, analysis, and topology, Contemp. Math., vol. 350, Amer. Math. Soc., Providence, RI, 2004, pp. 83-97.

[DP05] D. Danielli and A. Petrosyan, A minimum problem with free boundary for a degenerate quasilinear operator, Calc. Var. Partial Differential Equations (2005), 1-28, DOI: 10.1007/s00526-004-0294-5. 
[DSJ05] D. De Silva and D. Jerison, A singular energy minimizing free boundary preprint 2005.

[Eva82] L. C. Evans, A new proof of local $C^{1, \alpha}$ regularity for solutions of certain degenerate elliptic p.d.e, J. Differential Equations 45 (1982), no. 3, 356-373.

[IM89] T. Iwaniec and J. J. Manfredi, Regularity of p-harmonic functions on the plane, Rev. Mat. Iberoamericana 5 (1989), no. 1-2, 1-19.

[Lew83] J. L. Lewis, Regularity of the derivatives of solutions to certain degenerate elliptic equations, Indiana Univ. Math. J. 32 (1983), no. 6, 849-858.

[Man88] J. J. Manfredi, p-harmonic functions in the plane, Proc. Amer. Math. Soc. 103 (1988), no. $2,473-479$.

[Tru67] N. S. Trudinger, On Harnack type inequalities and their application to quasilinear elliptic equations, Comm. Pure Appl. Math. 20 (1967),721-747.

[Wei99] G. S. Weiss, Partial regularity for a minimum problem with free boundary, J. Geom. Anal. 9 (1999), no. 2, 317-326.

Department of Mathematics, Purdue University, West Lafayette, IN 47907

E-mail address: danielli@math.purdue.edu, arshak@math.purdue.edu 